\title{
Critical Temperature of an Interacting Bose Gas in a Generic Power-Law Potential
}

\author{
Luca Salasnich \\ Istituto Nazionale per la Fisica della Materia, Unità di Milano, \\ Dipartimento di Fisica, Università di Milano, \\ Via Celoria 16, 20133 Milano, Italy \\ E-mail: salasnich@mi.infm.it
}

\begin{abstract}
We investigate the critical temperature of an interacting Bose gas confined in a trap described by a generic isotropic power-law potential. We compare the results with respect to the non-interacting case. In particular, we derive an analytical formula for the shift of the critical temperature holding to first order in the scattering length. We show that this shift scales as $N^{\frac{n}{3(n+2)}}$, where $N$ is the number of Bosons and $n$ is the exponent of the power-law potential. Moreover, the sign of the shift critically depends on the power-law exponent $n$. Finally, we find that the shift of the critical temperature due to finite-size effects vanishes as $N^{-\frac{2 n}{3(n+2)}}$.
\end{abstract}


Nowadays more than twenty experimental groups have achieved Bose-Einstein condensation (BEC) in trapped alkali-metal atoms by using different geometries of the confining trap and atomic species. ${ }^{1-3}$ The experimental results on these dilute gases of weakly-interacting Bosons are in quite good agreement with the theoretical predictions both above and below the BEC critical temperature (for a recent review see Ref. 4).

An interesting problem is the behavior of the critical temperature $T_{c}$ as a function of the number $N$ of Bosons. Unfortunately, the experimental data of the critical temperature are not very precise but they seem to qualitatively confirm the predictions. In many experiments with alkali-metal atoms the trap can be described by a harmonic potential $U(\mathbf{r})=(1 / 2) m \omega^{2} r^{2}$. In this case, it is well known that in the large $N$ limit the critical temperature $T_{c}^{(0)}$ of an ideal gas scales as $N^{\frac{1}{3}}, 5$ while the shifts of the critical temperature due to the finite number of Bosons and the interaction scale as $N^{-\frac{1}{3}}$ and $N^{\frac{1}{6}}$, respectively. ${ }^{6,7}$ Note that the shape of the trapping potential plays a decisive role for the critical temperature, as we have recently shown with a dilute gas of hydrogen atoms in a Ioffe trap. ${ }^{8}$

An important class of trapping potentials is provided by power-law potentials $U(\mathbf{r})=$ $A r^{n}$. Power-law potentials have been proposed to cool the Bose gas in a reversible way by adiabatically changing the shape of the trap at a rate slow compared to the internal equilibration rate. ${ }^{9-11}$ For these potentials it has been shown that the critical temperature $T_{c}^{(0)}$ of an ideal Bose gas scales as $N^{\frac{2 n}{3(n+2)}}$ in a 3 -dimensional space ${ }^{5}$ and as $N^{\frac{2 n}{d(n+2)}}$ in a generic d-dimensional space. ${ }^{12}$

In this paper we extend previous results of an ideal Bose gas in power-law potentials. In particular, we study the role of the inter-atomic interaction and finite-size effects. We derive analytical formulas for the total number of particles and the critical temperature of BEC phase transition.

The starting point of our investigation is the semiclassical density profile $\rho^{(0)}(\mathbf{r})$ of an ideal Bosons in a trapping external potential $U(\mathbf{r})$. In the grand canonical ensemble, the density profile is given by 


$$
\rho^{(0)}(\mathbf{r})=\left(\frac{m k T}{2 \pi \hbar^{2}}\right)^{\frac{3}{2}} B_{3 / 2}\left(e^{(\mu-U(\mathbf{r})) / k T}\right),
$$

where $T$ the absolute temperature with $k$ the Boltzmann constant, $\mu$ is the chemical potential and

$$
B_{r}(z)=\sum_{i=1}^{\infty} \frac{z^{i}}{i^{r}}
$$

is the so-called Bose function. This result is the generalization of the formula for an ideal Bose gas in a box of volume $V$. It shows that, in the semiclassical limit, the nonhomogeneous formula is obtained with the substitution $\mu \rightarrow \mu-U(\mathbf{r})$, also called local density approximation. ${ }^{7,12}$

In the case of weakly-interacting Bosons, the previous formula must be substituted by the following one

$$
\rho(\mathbf{r})=\left(\frac{m k T}{2 \pi \hbar^{2}}\right)^{\frac{3}{2}} B_{3 / 2}\left(e^{(\mu-U(\mathbf{r})+2 g \rho(\mathbf{r})) / k T}\right),
$$

where $g=4 \pi \hbar^{2} a / m$ is the interaction coupling constant fixed by the s-wave scattering length $a$. This equation is a mean-field self-consistent equation that can be derived, within the semiclassical approximation and above the critical temperature $T_{c}$, from the BogolibovPopov equations, which describe the elementary excitations of the Bose gas. ${ }^{4,7}$ The chemical potential $\mu$ is fixed by imposing the following normalization condition

$$
N=\int \rho(\mathbf{r}) d^{3} \mathbf{r}
$$

where $N$ is the total number of Bosons. Note that below the critical temperature $T_{c}$, the density of the left-hand side in Eq. (3) describes only the non-condensed thermal cloud, while the density in the right-hand side is the total density, namely condensed and non-condensed density. Nevertheless, to calculate the BEC transition temperature $T_{c}$ it is sufficient the study the non-condensed thermal cloud. In fact, by imposing for the density profile (3) the normalization condition (4) one finds the total number of particles $N$ as a function of $T$ and $\mu$. At the critical temperature $T_{c}$, the chemical potential $\mu$ is minimum $(\mu=0$ in the large $N$ limit) and thus one gets the critical temperature $T_{c}$ as an implicit function of $N .{ }^{4-7,12}$ 
Let us consider a generic isotropic power-law potential given by

$$
U(\mathbf{r})=A r^{n}=\frac{1}{2}\left(\frac{\hbar \omega_{0}}{r_{0}^{n}}\right) r^{n}
$$

where $n$ is the power-law exponent and $A$ is the trap constant. Here we have introduced an energy parameter $\hbar \omega_{0}$ and a length parameter $r_{0}$, which can be chosen as $r_{0}=\left(\hbar /\left(m \omega_{0}\right)\right)^{1 / 2}$. By Taylor expanding the Eq. (3) to the first order in the coupling constant $g$ one finds

$$
\rho(\mathbf{r})=\rho^{(0)}(\mathbf{r})-\frac{2 g}{k T}\left(\frac{m k T}{2 \pi \hbar^{2}}\right)^{\frac{3}{2}} B_{1 / 2}\left(e^{(\mu-U(\mathbf{r})) / k T}\right) \rho^{(0)}(\mathbf{r})
$$

where $\rho^{(0)}(\mathbf{r})$ is the non-interacting density given by Eq. (1). Then, by imposing the normalization condition one gets

$$
N=\left(\frac{k T}{\hbar \omega_{0}}\right)^{\frac{3(n+2)}{2 n}} \frac{2^{\frac{3}{n}} \Gamma\left(\frac{3}{n}+1\right)}{2^{\frac{3}{2}} \Gamma\left(\frac{3}{2}+1\right)} B_{\frac{3(n+2)}{2 n}}\left(e^{\mu / k T}\right)\left[1-2 g\left(\frac{m}{2 \pi \hbar^{2}}\right)^{\frac{3}{2}}(k T)^{\frac{1}{2}} \frac{S_{\frac{3}{n}}\left(e^{\mu / k T}\right)}{B_{\frac{3(n+2)}{2 n}}\left(e^{\mu / k T}\right)}\right]
$$

where $\Gamma(r)$ is the Euler factorial function and

$$
S_{r}(z)=\sum_{i j=1}^{\infty} \frac{z^{i+j}}{i^{\frac{3}{2}} j^{\frac{1}{2}}(i+j)^{r}} .
$$

In Eq. (7) the total number $N$ of Bosons is a function of temperature $T$, chemical potential $\mu$ and interaction constant $g$, namely $N=N(T, \mu, g)$. Setting $g=0$ we have the number of particles for an ideal Bose gas. In this case, to find the critical temperature $T_{c}^{(0)}$ we put $\mu=0$ and obtain

$$
k T_{c}^{(0)}=c(n) \hbar \omega_{0} N^{\frac{2 n}{3(n+2)}},
$$

where $c(n)$ is a numerical coefficient,

$$
c(n)=\left[\frac{2^{\frac{3}{2}} \Gamma\left(\frac{3}{2}+1\right)}{2^{\frac{3}{n}} \Gamma\left(\frac{3}{n}+1\right) \zeta\left(\frac{3}{n}+\frac{3}{2}\right)}\right]^{\frac{2 n}{3(n+2)}}
$$

and $\zeta(r)=B_{r}(1)$ is the Riemann $\zeta$-function. As previously stated, this formula was obtained for the first time by Bagnato, Pritchard and Kleppner. ${ }^{5}$

To determine the effect of the inter-atomic interaction on the critical temperature, we expand the Eq. (7) around $\mu=0, T=T_{c}^{(0)}$ and $g=0$ :

$$
N(T, \mu, g)=N\left(T_{c}^{(0)}, 0,0\right)+\frac{\partial N}{\partial T}\left(T_{c}^{(0)}, 0,0\right)\left(T-T_{c}^{(0)}\right)+
$$




$$
+\frac{\partial N}{\partial \mu}\left(T_{c}^{(0)}, 0,0\right) \mu+\frac{\partial N}{\partial g}\left(T_{c}^{(0)}, 0,0\right) g .
$$

At the critical temperature $\left(T=T_{c}\right)$ of the interacting system, for large $N$, the chemical potential can be written $\mu=2 g \rho^{(0)}(\mathbf{0})$ as suggested by Eq. (3). ${ }^{4,7}$ In this way, from Eq. (1), Eq. (9) and Eq. (11) we get the main result of the paper

$$
\frac{T_{c}-T_{c}^{(0)}}{T_{c}^{(0)}}=-d(n)\left(\frac{a}{r_{0}}\right) N^{\frac{n}{3(n+2)}},
$$

where

$$
d(n)=\frac{2^{\frac{3}{2}}\left(\zeta\left(\frac{3}{n}+\frac{1}{2}\right) \zeta\left(\frac{3}{2}\right)-S\left(\frac{3}{n}\right)\right)}{\pi^{\frac{1}{2}} \zeta\left(\frac{3}{n}+\frac{3}{2}\right)\left(\frac{3}{n}+\frac{3}{2}\right)}\left[\frac{2^{\frac{3}{2}} \Gamma\left(\frac{3}{2}+1\right)}{2^{\frac{3}{n}} \Gamma\left(\frac{3}{n}+1\right) \zeta\left(\frac{3}{n}+\frac{3}{2}\right)}\right]^{\frac{n}{3(n+2)}},
$$

and $S(r)=S_{r}(1)$. An inspection of Eq. (12) shows that the shift due to the interaction can be either negative or positive, depending on the sign of the scattering length $a$ and of the coefficient $d(n)$. As shown in Tab. $1, d(n)$ diverges for $n=6$ and goes to zero as $n \rightarrow \infty$.

The temperature shift (12) scales as $N^{\frac{n}{3(n+2)}}$, where $n$ is the exponent of the power-law potential. It means that increasing $n$ one can amplify the shift of the critical temperature due to the interaction. This effect is shown in Fig. 1, where we plot the critical temperature $T_{c}$ as a function of the number $N$ of bosons for $n=2$ and $n=4$. It is important to observe that in our approach the shift is always associated with a change in the central density produced by inter-atomic forces. The relationship between the critical temperature and the corresponding value of the density in the center of the trap is unaffected by the interaction. Moreover, the shift vanishes for a homogeneous system where the density is uniform. Note that with $n=2$ one recovers the shift-formula obtained by Giorgini, Pitaevskii and Stringari for a dilute Bose gas in harmonic potential. ${ }^{7}$

Another interesting aspect of BEC in trapping potentials are finite-size effects. In an ideal gas, BEC starts at the temperature for which the chemical potential $\mu$ reaches the energy of the lowest solution of the Schrödinger equation. In the case of a power-law potential this energy is $\alpha_{n} \hbar \omega_{0}$, where $\alpha_{n}$ is a coefficient depending on the power-law exponent $n$. For example, in the case of the harmonic potential we have $\alpha_{2}=3 / 2$. In general, the coefficient 
$\alpha_{n}$ must be numerically evaluated and only in the large $N$ limit one can set $\mu=0$ at the transition temperature. We can estimate finite-size effects on the basis of Eq. (11) by setting $g=0, \mu=\alpha_{n} \hbar \omega_{0}$ and $T=T_{c}^{(F S)}$. In this way we obtain

$$
\frac{T_{c}^{(F S)}-T_{c}^{(0)}}{T_{c}^{(0)}}=-e(n) \alpha_{n} N^{-\frac{2 n}{3(n+2)}}
$$

where

$$
e(n)=\frac{\zeta\left(\frac{3}{n}+\frac{1}{2}\right)}{\zeta\left(\frac{3}{n}+\frac{3}{2}\right)\left(\frac{3}{n}+\frac{3}{2}\right)}\left[\frac{2^{\frac{3}{2}} \Gamma\left(\frac{3}{2}+1\right)}{2^{\frac{3}{n}} \Gamma\left(\frac{3}{n}+1\right) \zeta\left(\frac{3}{n}+\frac{3}{2}\right)}\right]^{-\frac{2 n}{3(n+2)}}
$$

Numerical values of the coefficient $e(n)$ are reported in Tab. 1. The temperature shift (14) originating from finite size effects changes sign as at $n=6$, where the coefficient $e(n)$ diverges, and, as expected, the shift vanishes in the large $N$ limit. In particular, with a fixed number $N$ of Bosons, one can strongly reduce this finite-size shift by increasing the power-law exponent $n$ as shown in Fig. 1, where we have set $\alpha_{4}=\alpha_{2}=3 / 2$. The finite-size shift predicted by Grossmann and Holthaus ${ }^{6}$ for a Bose gas in harmonic potential is obtained from Eq. (14) with $n=2$.

In conclusion, we have derived two remarkable formulas for the critical temperature of a weakly-interacting Bose gas in a generic isotropic power-law potential. One formula gives, to the first order in the scattering length, the shift of the critical temperature due to the inter-atomic interaction. The temperature shift is strongly enhanced by a large the power-law exponent but the sign of the shift depends in a non trivial way on the power-law exponent. The other formula shows that, as expected, the finite-size effects, namely setting the chemical potential equal to zero at the Bose-Einstein transition, are negligible in the large $N$ limit. Moreover, with a fixed number $N$ of bosons, the finite-size effects are reduced by a large power-law exponent. 


\section{REFERENCES}

1. M.H. Anderson, J.R. Ensher, M.R. Matthews, C.E. Wieman, and E.A. Cornell, Science 269 (1995) 189.

2. K.B. Davis, M.O. Mewes, M.R. Andrews, N.J. van Druten, D.S. Drufee, D.M. Kurn, and W. Ketterle, Phys. Rev. Lett. 75 (1995) 3969.

3. C.C. Bradley, C.A. Sackett, J.J. Tollet, and R.G. Hulet, Phys. Rev. Lett. 75 (1995) 1687.

4. F. Dalfovo, S. Giorgini, L.P. Pitaevskii, and S. Stringari, Rev. Mod. Phys. 71 (1999) 463.

5. V. Bagnato, D.E. Pritchard and D. Kleppner, Phys. Rev. A 35 (1987) 4354.

6. S. Grossmann and M. Holthaus, Phys. Lett. A 208 (1995) 108.

7. S. Giorgini, L. Pitaevskii and S. Stringari, Phys. Rev. A 54 (1996) 4633.

8. B. Pozzi, L. Salasnich, A. Parola and L. Reatto, Eur. Phys. Jour. D 11 (2000) 367.

9. W. Ketterle and D.E. Pritchard, Phys. Rev. A 46(1992) 4051.

10. P.W.H. Pinkse, A. Mosk, M. Weidenmuller, M.W. Reynolds, T.W. Hijmans, and J.T.M. Walraven, Phys. Rev. Lett. 78 (1997) 990.

11. D.M. Stamper-Kurn, H.J. Miesner, A.P. Chikkatur, S. Inouye, J. Stenger, and W. Ketterle, Phys. Rev. Lett. 81 (1998) 2194.

12. L. Salasnich, J. Math. Phys. 41 (2000) 8016. 


\begin{tabular}{|c|cccccccc|}
\hline & 1 & 2 & 3 & 4 & 5 & 6 & 7 & 8 \\
\hline$c(n)$ & 0.56 & 0.94 & 1.14 & 1.25 & 1.31 & 1.35 & 1.04 & 1.00 \\
$d(n)$ & 0.64 & 1.33 & -0.23 & 2.41 & 7.24 & $\infty$ & -41.57 & -42.96 \\
$e(n)$ & 0.40 & 0.50 & 0.29 & 1.11 & 2.45 & $\infty$ & -2.96 & -1.61 \\
$\frac{2 n}{3(n+2)}$ & $2 / 9$ & $1 / 3$ & $2 / 5$ & $4 / 9$ & $10 / 21$ & $1 / 2$ & $14 / 27$ & $8 / 15$ \\
$\frac{n}{3(n+2)}$ & $1 / 9$ & $1 / 6$ & $1 / 5$ & $2 / 9$ & $5 / 21$ & $1 / 4$ & $7 / 27$ & $4 / 15$ \\
\hline
\end{tabular}

TAB. 1. Numerical coefficients as a function of the power-law exponent $n$. In the limit $n \rightarrow \infty$ one gets: $c(n) \rightarrow 1.27, d(n) \rightarrow 0, e(n) \rightarrow-0.29,2 n /(3(n+2)) \rightarrow 2 / 3$, and $n /(3(n+2)) \rightarrow 1 / 3$. 


\section{FIGURES}

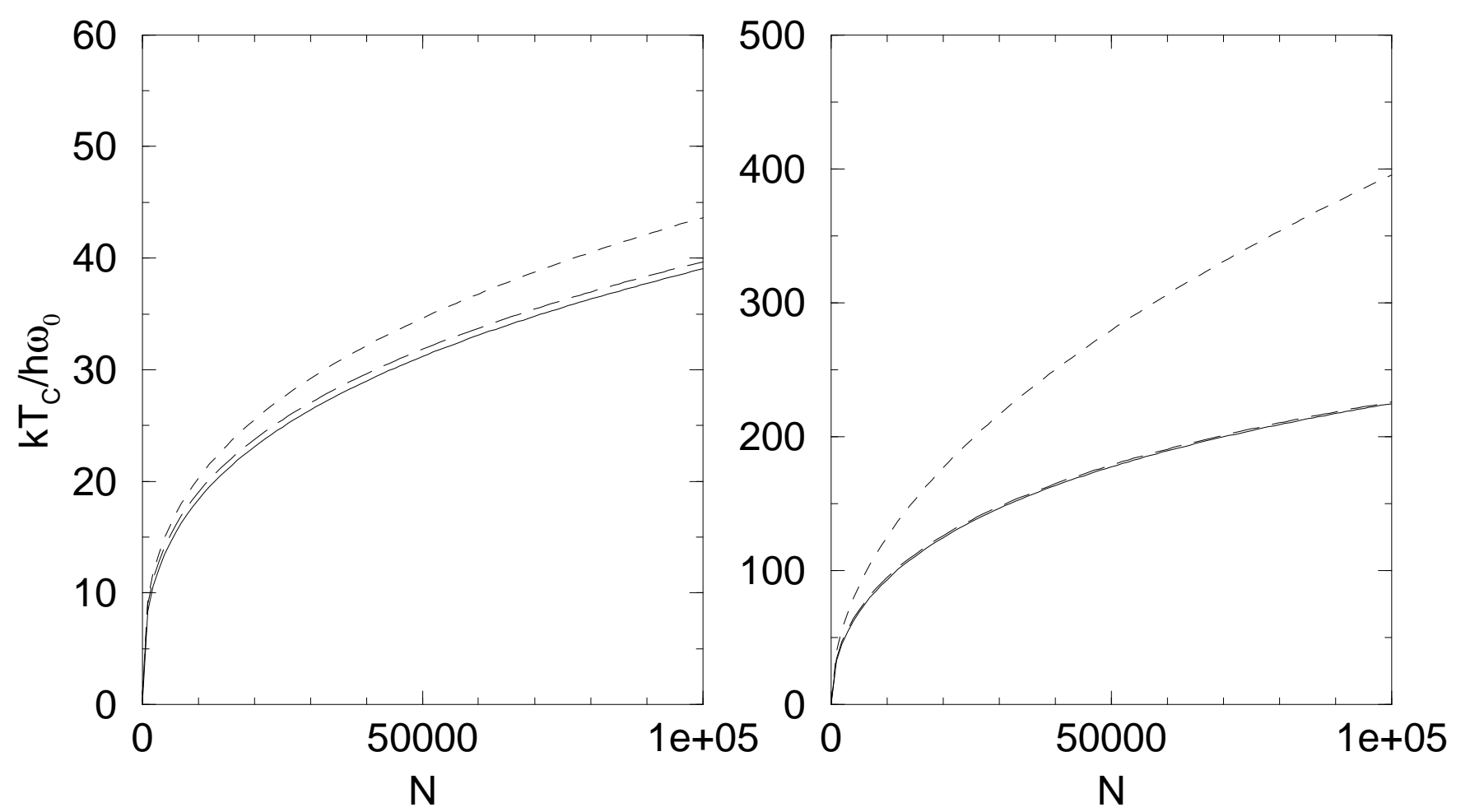

FIG. 1. Critical temperature $T_{c}$ as a function of the number $N$ of Bosons. Power-law exponent $n=2$ on left and $n=4$ on the right. Ideal Bose gas (dashed line), interacting Bose gas (long dashed line), interacting Bose gas with finite-size corrections (full line). Interaction strength $a / r_{0}=10^{-2}$. 